\title{
Probing a singlet scalar in electron- positron colliders
}

\section{Seddigheh Tizchang*}

School of Particles and Accelerators, Institute for Research in Fundamental Sciences (IPM) P.O.

Box 19395-5531, Tehran, Iran

E-mail: s.tizchang@ipm.ir

\section{Daruosh Haji Raissi}

Faculty of Sciences, Department of Physics, Ayatollah Amoli Branch, Islamic Azad University, Amol, Mazandaran, Iran

E-mail: daruoshraissi96@ipm.ir

\section{Mojtaba Mohammadi Najafabadi}

School of Particles and Accelerators, Institute for Research in Fundamental Sciences (IPM) P.O. Box 19395-5531, Tehran, Iran

E-mail: mojtabaeipm.ir

\begin{abstract}
We consider a simplified model containing a new neutral singlet scalar and a vector-like top quark, which mix with the Higgs boson and top quark of the SM, respectively. The presence of a vectorlike top quark in addition to a new scalar, which is common in many new physics models, is well motivated as they help stabilize the electroweak vacuum of the Higgs potential in the SM. Beside the theoretical motivations, this model can explain the observed enhancements in the di-Higgs production at the LHC experiments. We propose that the associated production of the new singlet scalar with a photon at future electron-positron colliders could be one of the practical channels to search for new physics. We compute the production rate of the scalar plus a photon at the proposed high luminosity electron-positron colliders such as ILC and CLIC.
\end{abstract}

The 39th International Conference on High Energy Physics (ICHEP2018)

4-11 July, 2018

Seoul, Korea

${ }^{*}$ Speaker. 
Our study focuses on a simplified model in which the Standard Model (SM) can be extended with a real neutral scalar and a vector-like quark with the same quantum numbers as the SM righthanded top quark called top partner which are well motivated to control the instability of electroweak vacuum [1]. Top partner (T) couples to SM particles through Yukawa type interaction terms. After the Spontaneous Symmetry Breaking (SSB), both the SM Higgs doublet and the new scalar will acquire non-zero vacuum expectation values (vevs): $v_{H}$ is the SM Higgs vev and $v_{S}$ is the singlet scalar vev which is expected to be larger than $v_{H}$. The scalar vev is used to give mass to the top partner. The scalar and the SM Higgs boson mix after SSB. Therefore, two new eigenstates are generated: the SM Higgs and the heavier scalar $h_{2}$. Moreover, top partner mixes only with the SM top quark where the lighter new eigenstate becomes the SM top quark. As a result, the scalar, Yukawa and gauge sector of the SM Lagrangian will be modified. The details of the SSB and mixing are given in Ref [2]. The model has five free parameters: the scalar vev $v_{S}$, the mass of new heavy scalar $m_{h_{2}}$, the mass of top partner $m_{T}$, the mixing angles of scalars $\theta$ and fermions $\theta_{L}$.

In this paper, we consider the heavy scalar production with a photon as a tool to constrain the free parameters of the simplified model. Similar to the SM Higgs plus photon production, $e^{-} e^{+} \rightarrow h_{2} \gamma$ is a loop-level process in which the top quark, $\mathrm{W}$ and $\mathrm{Z}$ bosons, electron and its neutrino contribute in triangle and box diagrams. There is additional contribution to the triangle loops coming from the top partner. The details of cross section calculation are available in Ref [3]. The cross section for the process $e^{-} e^{+} \rightarrow h_{2} \gamma$ as a function of different variables are shown in Fig.1. The left plot illustrates the dependence of the $d \sigma / d \cos \theta_{s}$ with respect to the scattering angle $\theta_{s}$, for different center of mass energies $\sqrt{s}=500 \mathrm{GeV}$ and $1,3 \mathrm{TeV}$. The middle plot shows the total cross section as a function of $\sqrt{s}$ for two benchmarks: $\left(v_{S}, m_{T}\right)=(400,750)$ and $(350,1000) \mathrm{GeV}$. The cross section is maximum at $T \bar{T}$ threshold $\sqrt{s}=2 m_{T}$. The cross section in terms of new scalar mass for three values of $\sqrt{s}$ is presented in the right plot. All the plots indicate that cross section varies according to the sign of the scalar mixing angle. That is because the cross section is proportional to an odd power of $\sin \theta$. Although the cross section for this process is rather small, the presence of a hard photon in the final state makes this channel a clean probe to hunt the new scalar in high luminosity electron-positron colliders.
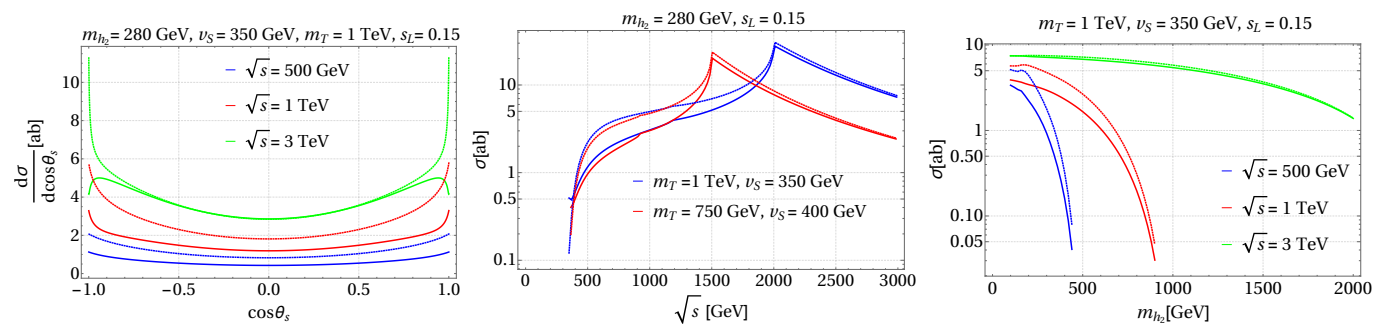

Figure 1: The cross section for $e^{-} e^{+} \rightarrow h_{2} \gamma$ as a function of (Left) scattering angle, (middle) center of mass energy and (right) scalar mass, with $s_{\theta}=0.15$ (solid) and $s_{\theta}=-0.15$ (dotted).

\section{References}

[1] M. L. Xiao and J. H. Yu, Stabilizing electroweak vacuum in a vectorlike fermion model, Phys. Rev. D 90 (2014) no.1, 014007 [hep-ph/1404.0681].

[2] M. J. Dolan, J. L. Hewett, M. Krämer and T. G. Rizzo, Simplified Models for Higgs Physics: Singlet Scalar and Vector-like Quark Phenomenology, JHEP 1607 (2016) 039 [hep-ph/1601.07208].

[3] D. H. Raissi, S. Tizchang and M. Mohammadi Najafabadi, Loop induced singlet scalar production through the vector like top quark at future lepton colliders, [hep-ph/1810.08797]. 